\title{
QUASICRYSTALS THE STATE OF THE ART
}


This page is intentionally left blank 
Directions in Condensed Matter Physics - Vol. 11

\title{
QUASICRYSTALS THE STATE OF THE ART
}

\author{
Editors \\ D P DiVincenzo \\ IBM T. J. Watson Research Center \\ Yorktown Heights, NY \\ P J Steinhardt \\ Department of Physics \\ University of Pennsylvania
}




\section{Published by}

World Scientific Publishing Co. Pte. Ltd.

P O Box 128, Farrer Road, Singapore 9128

USA office: Suite 1B, 1060 Main Street, River Edge, NJ 07661

UK office: 73 Lynton Mead, Totteridge, London N20 8DH

\section{Cover Design}

Inset: Scanning Tunneling Microscopy of Quasicrystal Surface provided by A. Kortan (AT\&T Bell Labs.)

Abstract Design: Chiral Smectic Quasicrystal Pattern provided by

T. Lubensky (University of Pennsylvania)

\section{QUASICRYSTALS: THE STATE OF THE ART}

- Directions in Condensed Matter Physics Vol. 11

Copyright O 1991 by World Scientific Publishing Co. Pte. Ltd.

All rights reserved. This book, or parts thereof, may not be reproduced in any form or by any means, electronic or mechanical, including photocopying, recording or any information storage and retrieval system now known or to be invented, without written permission from the Publisher.

ISBN 981-02-0522-8

ISBN $981-02-0523-6 \mathrm{pbk}$

Printed in Singapore by JBW Printers \& Binders Pte. Ltd. 


\section{Table of Contents}

Chapter 1: Progress and Current Issues in Quasicrystals

D. P. DiVincenzo and P. J. Steinhardt

1. The Basics

2. Early Developments in Experiment 2

3. Early Developments in Theory 3

4. The State of the Art 8

Chapter 2: Order and Disorder in Icosahedral Alloys 17 P. A. Bancel

1. Introduction 17

2. Phason Disorder 18

3. Face Centered Icosahedral Alloys 23

4. The AlCuFe Phase Diagram 26

5. Transformations of the Icosahedral Phase 29

6. Conclusions 47

Appendix $\quad 52$

References $\quad 54$

Chapter 3: Neutron Scattering Approaches to Quasicrystals 57 $C h$. Janot and M. de Boissieu

1. Introduction 57

2. From Diffraction Patterns to Atom Positions:

3. Some Experimental Achievements in Quasicrystals Structures 72

$\begin{array}{ll}\text { 4. Conclusions } & 91\end{array}$

References $\quad 93$

Chapter 4: High-Resolution Electron Microscopy of Quasicrystals 95 K. Hiraga

1. Introduction 95

2. Icosahedral Quasicrystals 95

3. Decagonal Quasicrystals $\quad 100$

4. Conclusions 108

References $\quad 110$ 
Chapter 5: Scanning Tunneling Microscopy Studies of Quasicrystals R. S. Becker and A. R. Kortan

1. Introduction

2. Experimental Results

3. Structure Modelling Based on STM

4. Comparison with Models Based on Bulk Studies

5. Conclusions

References

Chapter 6: (Quasi) Crystallography is Better in Fourier Space N. D. Mermin

1. Generalized Crystallography in Fourier Space

2. Computation of the Cubic and Icosahedral Space Groups

Chapter 7: Matching Rules for Quasicrystalline Tilings

$K$. Ingersent

1. Introduction

2. Tiling Basics

3. Matching Rules

4. Systematic Analysis of Matching Rules for Two-Dimensional Tilings

5. Matching Rules for Three-Dimensional Tilings

6. The Physics of Matching Rules

Chapter 8: Growth Rules for Quasicrystals

J. E. S. Socolar

1. Introduction

2. Phason Disorder and Random Accretion Models

3. Local Rules for Growing Penrose Tilings

4. Generalization to Other Tilings and Symmetries

5. Imperfect Application of the Rules

6. Icosahedral Growth: Open Questions 
Chapter 9: Continuous Atomic Surfaces

L. S. Levitov

1. Introduction

2. Continuous Atomic Surfaces: General Considerations

3. Continuous Atomic Surfaces for Icosahedral Symmetry

4. Five-, Eight- and Twelve-fold Symmetries

Chapter 10: Chiral Smectics as Quasicrystals

T. C. Lubensky, S. R. Renn and T. Tokihiro

1. Introduction

275

2. How to Twist a Smectic

277

3. The de Gennes Model and the Superconductor Analogy 286

4. Lock-in to the Commensurate Quasicrystal 292

5. Conclusions

304

References

Chapter 11: Experimental Studies of Electronic Transport in Quasicrystals

K. Kimura and S. Takeuchi

1. Introduction

2. Icosahedral Phases Consisting Only of Simple Metals

3. Nonmagnetic Icosahedral Phases Including Transition Metals

4. Icosahedral Phases Involving Magnetic Moments

5. Decagonal Phases

6. Concluding Remarks

Appendix

References

Chapter 12: Electronic Structure and Transport of Quasicrystals

T. Fujiwara and H. Tsunetsugu

1. Introduction

2. One-dimensional Quasicrystals

3. Two-dimensional Quasicrystals

4. Three-dimensional Quasicrystals

5. Localization and Electron Transport

355

6. Conclusions

References 
Chapter 13: Electronic-Structure and Total-Energy Calculation for Quasicrystals and Related Crystals

A. E. Carlsson and R. Phillips

1. Introduction

2. Small-Cluster Calculations 364

3. Related Periodic Systems $\quad 370$

4. Explicit Calculations for Quasicrystalline Structures 384

5. Summary and Future Prospects 397

$\begin{array}{ll}\text { References } & 398\end{array}$

Chapter 14: Faceting and Surface Roughening in Quasicrystals 403 T.-L. Ho

1. Introduction: The Curious Quasicrystal Facets 403

2. Brief Experimental Survey 404

3. Quasicrystal Shapes at $T=0 \quad 406$

4. Roughening of Quasicrystal Surfaces 417

References $\quad 426$

Chapter 15: Random Tiling Models $\quad 429$

C. L. Henley

1. Introduction

2. Defining Tilings 433

3. Perpendicular Space Concepts $\quad 437$

4. Random Tilings 445

5. Random Tiling Hypothesis and Phases 452

6. Perp Space Fluctuations $\quad 460$

7. Diffraction 468

8. Calculational Methods $\quad 479$

9. Results 491

10. Random Tilings and Real Quasicrystals: Energy Terms 496

11. Conclusions $\quad 512$

References $\quad 518$ 